\title{
From Euclidean to fractal geometry: The case of disintegration of polyaniline colloid particles
}

\author{
B.M. Mandal \\ Polymer Science Unit, Indian Association for the Cultivation of Science, Jadavpur, \\ Calcutta 700 032, India.
}

\begin{abstract}
Spherical or oval shaped polyaniline colloid particles (ca. $50-100 \mathrm{~nm}$ ) prepared by oxidative dispersion polymerization of aniline proved to be compound particles; being composed of particulates (ca. $5 \mathrm{~nm}$ ). These compound particles can be disintegrated into the particulates in some situations with the help of ultrasound. The particulates being unstable undergo aggregation to yield fractal aggregates. The fractal dimensions (D) have been determined from the transmission electron microscopic images of the aggregates. The values of $D$ fit with a cluster-cluster aggregation model for the aggregates. The importance of the phenomenon in preparing conducting composites with low percolation threshold is discussed.
\end{abstract}

\section{INTRODUCTION}

Fractal objects are encountered in various fields of chemistry e.g. electrodeposition of metals (ref. 1-2) aggregation of colloid particles (ref. 2-7), carbon-black aggregates (ref. 8), randomly coiled polymer chain (ref. 9) etc. Fractals often have shapes and structures like dendrites. Although these objects appear irregular shaped, they have the important characteristics of self- similarity (ref. 10-11). Fractal structures are more open than Euclidian ones so that the former grow faster than the latter. Fractal aggregation is common amongst destabilised colloidal particles (ref. 3-7).

In this article we discuss that stable polyaniline colloid particles which are spherical or oval shaped Euclidian objects can be broken down to their constituent primary particles which being unstable undergo aggregation to fractal structures. This process of generating fractal structures which are electrically conducting may be profitably used to prepare conducting composites with low percolation thresholds.

\section{EXPERIMENTAL}

Polyaniline colloid particles were prepared by dispersion polymerization of aniline in water or aqueous ethanol using poly(vinyl methyl ether) (PVME) (ref. 12) $\left(\mathrm{M}_{\mathrm{v}}=52,000\right)$ or an oligo(vinyl ethyl ether) (OVEE) (ref. 13) $\left(M_{n}=1400\right)$ as stabilizers.

The particles were isolated by centrifugation and cleaned by redispersion in acidic aqueous ethanol followed by centrifugation. The cycle is repeated two more times.

For making composites the isolated PANI colloid particles were suspended in solutions of polymers using solvents which are compatible with the stabilizer used in the preparation of the PANI colloids. The suspension is then sonicated using an ultrasonics cleaning bath. The redispersions thus obtained were poured into petridishes and the solvents were evaporated off yielding composite films (ref. 14). The electrical conductivity of the films was measured using the van der Pauw method.

The morphology of the particles and the films were studied by transmission electron microscopy (ref. 1214).

\section{DISCUSSION}

It has been shown in our earlier paper that oval shaped polyaniline particles $(250 \times 190 \mathrm{~nm})$ prepared using PVME stabilizer disintegrate into nanoparticles $(<20 \mathrm{~nm})($ ref. 14$)$. These nanoparticles are however 
unstable. They self-assemble to produce 3-dimensional network structures even at very low concentrations. The percolation threshold for such composites turned out to be exceptionally low ca. 0.03 volume percent.

Earlier Armes studied the surface characteristics of PANI as well as polypyrrole particles by scanning tunneling microscopy (ref. 15). They concluded that the 100-200 nm PANI or PPy colloid particles are composed of much smaller particulates $(5$ to $10 \mathrm{~nm}$ ). Subsequently Banerjee obtained TEM pictures of OVEE stabilized PANI particles which confirmed the above observation (ref. 13). The inner structure of the colloid particles is also in accord with the dispersion polymerization mechanism proposed by Paine (ref. 16). The particulates that are produced at the onset of phase separation in dispersion polymerization may be identified with the nuclei which reduce their surface energy by way of aggregation with themselves bringing their bare surfaces in contact and perhaps sharing the adsorbed stabilizer. Some of the adsorbed stabilizer may also get dislodged from the particulates due to the stress generated through the Brownian collision of the particulates (ref. 17) and find their way on the particle surface while others get trapped inside the particles. The aggregates of nuclei soon find their surface covered with stabilizer molecule and thus stable particles form. The aggregation of nuclei with themselves (homocoalescence) ceases when the aggregates become stable particles (ref. 16). The latter grow subsequently by absorption of the nuclei (heterocoalescenec) (ref.16-18). However, since the polymer is semicrystalline, the aggregation process does not lead to coalescence of the particulates into a single coherent mass like that happens in the coalescence of liquid drops. Instead, the particles retain the discrete particulates which are weakly bonded through the stabilizer trapped in between the particulates. Sonication process dislodges the particulates and separates them from each other regenerating them in the system. The regenerated particulates however do not have the same surface properties as they had when they went on to produce the particles by aggregation.

It has been established from the studies of adsorption of polymers on solid surfaces that a polymer molecule undergoes reorientation after being adsorbed (ref. 19). Long ageing times are required to obtain the equilibrium state. It is therefore expected that the surface properties of the dislodged particulates will be different than when they assembled to form a particle or enter into a stable particle. Their aggregation pattern may therefore be different. Particle formation process yields a regular Euclidian object like spheres, ovals, rods etc. while disintegration followed by reaggregation yields irregular objects which have been proved to be fractal. The fractal dimension have been determined from an analysis of the TEM images of the aggregates. A value of 1.69 has been determined for the fractal dimension (D) (ref. 20). Earlier, Weitz and Oliveria determined fractal dimensions of aggregates formed in destabilised gold sols by way of analyzing the TEM images of the aggregates (ref. 4). They determined a value of $\sim 1.75$ for $D$ which is in agreement with the value of $1.8 \pm 0.1$ predicted from computer simulations of structures in three dimension when single particles as well as clusters of particles are allowed to diffuse and clusters grow by clustercluster as well as by cluster-particle contact (ref. 21).

\section{REFERENCES}

1. a) M. Matsushita, M. Sano, Y. Hayakawa, H. Honjo and Y. Sawada. Phys. Rev. Lett. 53, 286 (1984).

b) M. Matsushita, Y. Hayakawa and Y. Sawada. Phys. Rev. A. 32, 3814 (1985).

2. H. Kaufmann, A. I. Nazzal and O. R. Melroy. Phys. Rev. B. 35, 1881 (1987).

3. R. Forrest and T. A. Witten. Jr. J. Phys. A. 12, L109 (1979).

4. A. Weitz and M. Oliveria. Phys. Rev. Lett. 52, 1433 (1984).

5. Aubert and D. S. Cannell. Phys. Rev. Lett. 56, 738 (1986).

6. Y. Lin, H. M. Lindsay, D. A. Weitz, R. Klein, R. C. Ball and P. Meakin. J. Phys. Conden. Matter. 2, 3093 (1990)

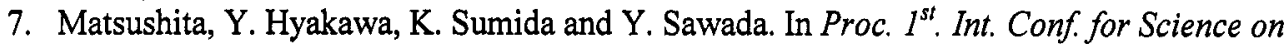
Form (S. Ishizaka, ed.), p. 23, KTK Scientific, Tokyo (1986).

8. R. Herd, G. V. McDonald and W. M. Hess. Rubber Chem. Tech. 65, 107 (1992).

9. Zallen, The Physics of Amorphous Solids, chap. 3. John Wiley, New York, 1983.

10. B. Mandelbrot. The Fractal Geometry of Nature, Freeman, New York (1983).

11. P. Kadanoff. Physics Today. 39(no. 2), 6 (1986).

12. P. Banerjee, M. L. Digar, S. N. Bhattacharyya and B. M. Mandal. Eur. Polym. J. 30, 499 (1994).

13. P. Banerjee. Ph. D. Thesis, Calcutta University (1995). 
14. P. Banerjee and B. M. Mandal. Macromolecules. 28, 3940 (1995).

15. P. Armes, M. Aldissi, M. Hawley. J. G. Berry and S. Gottesfeld. Langmuir. 7, 1447 (1991).

16. A.J. Paine. Macromolecules. 23, 3109 (1990).

17. H. Napper. Polymeric Stabilization of Colloidal Dispersions, p. 28. Academic Press, London (1983).

18. T.K. Mandal and B.M. Mandal. Langmuir. 13, 2421 (1997).

19. M. Schneider and S. Granick. Macromolecules. 25, 5054 (1992).

20. D. Chattopadhyay, S. Banerjee, D. Chakravorty and B. M. Mandal, Paper submitted for publication.

21. P. Meakin. Phys. Rev. A. 29, 997 (1984). 